\title{
9. Atracción mutua: Estudio sobre los maximadores enteramente, completamente, totalmente y absolutamente en combinación con adjetivos y participios
}

Johan Falk

Stockholms universitet

\section{Introducción}

El principio de la sedimentación de las colocaciones (entrenchment) ha cobrado importancia en los estudios lingüísticos de las últimas décadas. Las palabras se atraen formando conjuntos que parecen ser prefabricados en mayor o menor grado. La gramática construccional (Östman \& Fried 2005) y la teoría de las secuencias formulaicas (Wray 2002; Moreno Teva 20I2) han enfatizado el carácter aglutinador del lenguaje. Los enunciados no son combinaciones libres y aleatorias de elementos, sino que consisten en bloques que se rescatan enteros de la memoria. La expresión empleada más arriba, en mayor o menor grado, es un ejemplo típico de una secuencia fija. Es perfectamente natural decir en elevado grado y en gran medida, pero no se pueden intercambiar los adjetivos. Del mismo modo, en pleno día no se puede variar con los adjetivos claro, gran, lleno, total. El lenguaje tiende a formar colocaciones que pasan a ser fijas o semifijas.

Una de las preguntas que se plantea en este estudio es si los modificadores de grado máximo enteramente, completamente, totalmente y absolutamente (en adelante Max) forman pareja con los adjetivos y participios con los que combinan. Partimos del supuesto de que los sesgos distributivos -si los hay- están motivados por factores semánticos, es decir, por el modo en que se conceptualiza, por un lado, la gradación del Max y, por otro, el contenido semántico de los adjetivos y participios. Los Max estudiados aquí se distinguen de otros modificadores de

Cómo citar este capítulo:

Falk, Johan, Atracción mutua: Estudio sobre los maximadores enteramente, completamente, totalmente y absolutamente en combinación con adjetivos y participios. In: Engwall, Gunnel \& Fant, Lars (eds.) Festival Romanistica. Contribuciones lingüísticas - Contributions linguistiques - Contributi linguistici - Contribuições linguísticas. Stockholm Studies in Romance Languages. Stockholm: Stockholm University Press. 2015, pp. I75-I97. DOI: http://dx.doi.org/IO.I6993/bac.i. License: CC-BY 
grado como poco, bastante, muy y extremadamente ${ }^{\mathrm{I}}$ pero, además, se supone que la colocación de los Max con distintos grupos de adjetivos/ participios no es aleatoria. Se dice sin más absolutamente imposible, pero no se dice normalmente enteramente imposible ni extremadamente imposible. Al parecer, existen caminos trillados donde se atraen mutuamente el modificador y el modificado.

Nos interesa primero explorar las restricciones a que están sometidos los Max, segundo aclarar si existe cierta atracción mutua ${ }^{2}$ entre los cuatro Max, tomados individualmente, y grupos de adjetivos/participios. A nuestro juicio, se trata de explicar el acoplamiento de formas de conceptualizar más que constatar meramente la colocación de modificadores y adjetivos/participios específicos. Un concepto clave para explicar tendencias y sesgos es el de consonancia semántica. Con este término se entiende que dos constituyentes, unidos en una construcción endocéntrica, deben ser compatibles, es decir, no debe haber en la estructura semántica de los constituyentes elementos que generen una contradicción o una anomalía. Así, se dice mucha leche y no muchas leche(s) porque leche es un sustantivo de masa no contable, que no se combina con un adjetivo de pluralidad (muchas). El concepto de consonancia semántica se usa aquí de forma gradual, lo que significa que puede haber mucha o poca consonancia entre los elementos.

\section{Antecedentes, objetivo e hipótesis}

La modificación de grado ha sido objeto de varios estudios en inglés, principalmente Bierwisch (I967), Leech (I974), Cruse (I986), Allerton (I987) y Paradis (I997), en los que se inspira este estudio. Una fuente importante ha sido el estudio de Erman (2008) que plantea el empleo de los maximadores desde un punto de vista de la sedimentación (entrenchment), es decir su carácter formulaico. La estructura semántica del inglés y del español son fundamentalmente similares en el aspecto de gradación. Completely y completamente expresan una alta conformidad en frases como He is completely bald y Está completamente calvo. El problema tratado en los estudios citados concierne nuestra facultad de conceptualizar la forma de graduar distintos tipos de cualidades y condiciones y, así, es sobre todo un problema general y no exclusivo del inglés o del español. Esto no impide que un estudio paralelo en español pueda revelar usos idiosincrásicos de este idioma. 
Este estudio pretende responder a la siguiente serie de preguntas que plantea el uso y el significado de los Max de grado:

- ¿En qué consiste la especificidad de los Max con respecto a otros modificadores de grado? > Está completamente /?muy calvo. // Es muy l??totalmente generoso.

- ¿Qué rasgos debe poseer un adjetivo/participo para que se pueda ser modificado por un Max? > Es muy / ?absolutamente útil. // Es absolutamente l?muy inútil.

- ¿De qué modo la naturaleza de los Max condiciona la combinatoria con adjetivos y participios? > Está completamente lleno. // "Es completamente rico. ${ }^{3}$

- ¿Qué efectos semánticos y pragmáticos se producen si un Max transgrede los requisitos del adjetivo/participio? > ??Felipe es/está completamente soltero. // ??Felipe es muy soltero. ${ }^{4}$

¿Existe "una atracción mutua" entre determinados Max y determinados tipos de adjetivos/participios? Si es así, se puede hablar de una tendencia a crear colocaciones preferidas.

En el fondo, estas preguntas pueden ser reducidas a una interrogante común, que gira en torno al concepto de consonancia semántica. El objetivo del estudio es aclarar qué factores conceptuales rigen la consonancia semántica, permitiendo ciertas combinaciones, obstruyendo o excluyendo otras. Como se verá, el fenómeno de la modificación de grado no es, sin embargo, una cuestión de blanco o negro. En muchos casos se trata de tendencias y de combinaciones preferidas sin que se excluyan otras. Si completamente lleno resulta ser más natural (y posiblemente más frecuente) que muy lleno, esto no significa que haya un bloqueo total. Así, aspiramos no solo a ver los pasos lícitos y no lícitos del sistema, sino también lo que es atracción y ha dado lugar a usos sedimentados (entrenched uses). También en este último caso debe haber motivos para que una colocación se sedimente antes que otra. Por ejemplo, si absolutamente imposible es una combinación más frecuente que enteramente imposible (que sin duda se podría decir ${ }^{5}$ ) debe haber una razón de ello. Así, la noción de distribución sesgada es fundamental si se estudia la atracción mutua entre modificadores y elementos modificados.

El objetivo incluye un aspecto que nos parece relevante para explicar las combinatorias. Si existen sesgos en las distribuciones, será interesante estudiar el significado originario de los Max. Mantenemos la 
hipótesis de que los significados de entero, completo, total y absoluto -si bien se recubren en alto grado-, influyen en las colocaciones. Las imágenes esquemáticas de la sección 7 son elaboradas para apoyar la hipótesis de que los Max tienen distinta base conceptual.

No hay que descartar el problema del efecto modalizador de los Max. Decir que algo es absolutamente correcto no solo es una indicación de grado máximo, sino que revela una actitud subjetiva y una intención de convencer al interlocutor de la veracidad del aserto. Las palabras que indican grado máximo llevan a menudo una impronta modal y subjetiva.

\section{Método y materiales}

Este estudio se basa en un acopio de instancias de los cuatro maximadores extraídas de CREA ${ }^{6}$. Una recolección realizada entre I 996-2000 (todos los medios) arroja 8.408 ocurrencias, que ha sido el material de partida del que se han excluido los casos no pertinentes. Se ha formado un corpus de 723 ejemplos de Max + adjetivo/participio, que es el punto de partida de distintos tipos de recuentos y de un análisis más bien cualitativo.

Se ha calculado primero el número de lexemas modificados que son compartidos por los cuatro Max y, en un segundo paso, los lexemas exclusivos de los modificadores. Otro cálculo, que apunta a revelar la afinidad entre los Max, ha consistido en ver el número de lexemas compartidos por constelaciones de dos Max (en total 6 combinaciones).

El estudio es tanto cuantitativo como cualitativo. Es cuantitativo en el sentido de que se aspira a determinar frecuencias relativas, grado de solapamiento de los Max, grado de unicidad de ciertas combinaciones. Asimismo, lo cuantitativo es la base de análisis cualitativos que aspiran a descubrir y explicar ciertas distribuciones sesgadas. En particular será de interés ver si existe algún rastro del significado originario de los Max que pueda explicar las preferencias de las colocaciones.

\section{La modificación de grado y los Max}

Los Max estudiados aquí forman parte del dominio más amplio de la modificación de grado. Paradis (I997) refiere varias aproximaciones al estudio de los modificadores de grado, entre otras la de Bierwisch (I967), Leech (I974), Quirk et al. (I985), Cruse (I986) y Allerton (I987). 
La clasificación presentada por Quirk et al. (I985) se basa mayormente en la fuerza de la modificación que, por un lado, abarca AMPLIFIERS, que incluye 'boasters' y 'maximizers', y, por otro, DOWNTONERS, que a su vez incluyen tres categorías. Los 'boasters' son en principio palabras como muy, bastante, terriblemente, extremadamente, que sirven para ponderar una cualidad, una condición o una acción. Para delimitar el grupo de los 'maximizers', se debe pues precisar la diferencia entre los 'boasters' y los 'maximizers'.

La clasificación propuesta por Allerton (1987: 20) parte de la compatibilidad de los modificadores de grado con distintas clases de adjetivos, destacando su función semántica. Aplicadas al español, las clases son las siguientes:

- Modificadores escalares (poco, bastante, muy, extremadamente)

- Modificadores télicos que sirven para indicar grado con respecto a cierto propósito o norma (casi, apenas, solamente, perfectamente)

- Modificadores absolutos (totalmente, absolutamente, enteramente, del todo, completamente)

- Modificadores diferenciales que expresan la diferencia de grado entre un elemento cualitativo y un punto de referencia (un poco, mucho, un montón, demasiado, ligeramente).

Sin entrar en detalles de esta clasificación, no parece ser siempre fácil distinguir la clase b) de la clase d). En ambos casos los modificadores expresarían el desvío de una norma, función que se presenta también en los modificadores escalares (clase a). Decir que un objeto 'es barato', supone una norma para distinguir lo barato de lo no barato referente a cierta clase de objetos. El valor "plus" (caro) o "menos" (barato) con respecto a una escala de precios se refiere necesariamente a una norma conceptualizada.

Para nuestro propósito, lo más interesante es la distinción entre los modificadores escalares a) y los modificadores absolutos c). La categoría c) sería, según Allerton, 'superlative' y corresponde a los 'maximizers' de Quirk et al. (I985: 589 y sigs.) y Erman (2008). Mientras que los escalares indican cierto grado de una cualidad o condición que va de lo bajo a lo alto como en:

(I) poco inteligente $>$ bastante inteligente $>$ muy inteligente $>$ extremadamente inteligente,

los Max marcan un grado absoluto, es decir un punto que no se puede sobrepasar. Así, completamente expresa un grado que se encuentra en 
un extremo y que no se puede relativizar. Antes de continuar el análisis de los Max, volveremos al concepto de lo escalar.

Los modificadores referidos a escalas ("más o menos") como en (I) indican una posición relativa en un espacio cualitativo sin indicar de por sí una gradación cuantificada ni unos valores extremos fijos. Simplemente, extremadamente caro es más que muy caro, que es más que bastante caro, que es más un poco caro. Evidentemente, las proposiciones El agua está muy caliente y El agua está bastante fría se pueden objetivar mediante la escala termométrica, p.ej. lo caliente empieza en 25 grados C, pero esto no tiene nada de absoluto ya que la calificación del grado de calor o frío depende enteramente del contexto y de las decisiones tomadas7. Llamamos a este tipo de modificadores, modificadores de grado escalar (Moge).

Volviendo al término de 'boaster', se debe advertir que los modificadores de escalas no sólo indican cierto grado sino que sirven para ponderar el enunciado y darle un carácter subjetivo. El enunciado:

(2) Eso es mucha verdad,

no es primordialmente una gradación de la verdad sino una manera de abundar en la propia opinión y, tal vez, de reforzar la relación con el interlocutor.

Como veremos, los Max ('absolutive modifiers' de Allerton I987) seleccionan otra clase de adjetivos y participios que los modificadores escalares. El Max expresa un grado de una propiedad que no se puede sobrepasar. Al decir La piscina está completamente llenal vacía, damos a entender que hay un límite máximo y mínimo que se ha alcanzado. Con este tipo de modificador de grado no hay "más o menos", simplemente se dice que se ha alcanzado un límite extremo. La diferencia entre lo escalar y lo maximado queda claro en los siguientes ejemplos:

(3) Julián es muy tonto.

(4) Allí estaba, completamente atontado.

Es evidente que uno puede ser más o menos tonto y siempre puede haber una persona que sea aún más tonta que la que es muy tonta. En cambio el participio atontado refiere a un proceso que ha culminado en un estado definitivo.

Como se ha dicho, este estudio se centra en la categoría de los Max, que se irá cotejando con los Moge. Veremos ahora más en detalle la 
otra cara de la moneda, que es el modo en que los adjetivos y participios conceptualizan la gradación escalar y máxima. Esta es la pista que seguiremos para explicar las combinatorias y la eventual atracción mutua.

\section{Los adjetivos y los participios}

\subsection{Clasificación}

La siguiente clasificación de los adjetivos y los participios pretende recoger características que son relevantes para la modificación de grado y, particularmente, para delimitar el grupo de los $M a x^{8}$ :

Tabla 1a. Clases de adjetivos graduables

\begin{tabular}{lll}
\hline Adjetivos relativos (graduables) & \\
\hline Graduables plenos & Graduables limitados & \\
\hline Clase I & Clase 2 & Clase 3 \\
Adjetivos escalares & Adjetivos de límite & Adjetivos extremos \\
\hline caro - barato & lleno - vacio & maravilloso, insoportable \\
alto - bajo & intacto - destruido & \\
\hline
\end{tabular}

Tabla 1b. Clases de adjetivos no graduables

\begin{tabular}{ll}
\hline Adjetivos absolutos (no graduables) & \\
\hline Clase 4 & Clase 5 \\
Adjetivos invariables & Adjetivos relacionales \\
\hline mesa cuadrada, & reforma eclesiástica, \\
chico soltero & campaña presidencial \\
\hline
\end{tabular}

La distinción entre estos cinco tipos tiene que ver con el modo en que se pueden graduar. Descartamos desde el inicio las clases 4 y 5 que en principio no permiten ser graduados, salvo que se empleen con una intención específica y se traspasen a otra categoría. Un chico muy soltero da sentido si se extraen ciertas notas cualitativas próximas a "que se comporta como un típico soltero u otra calificación propia de los solteros”. 


\subsubsection{Clase 1}

La clase I agrupa a los adjetivos que representan la cualidad en una escala que va de menos a más. La mayoría de estos adjetivos forman parejas antonímicas y se sitúan en polos opuestos y extremos. Caro barato, joven - viejo y alto - bajo cubren una escala que permite graduación mediante poco, bastante, muy:

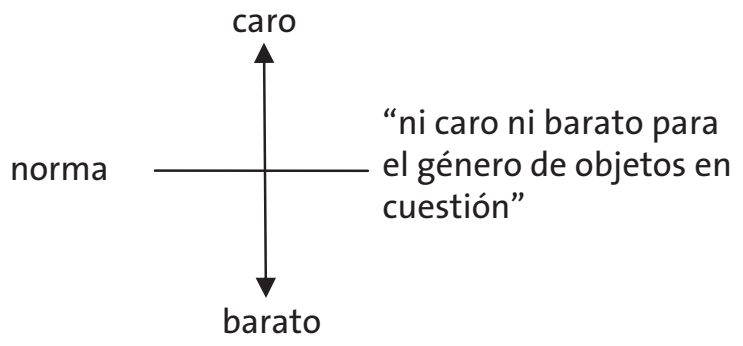

Los adjetivos de la clase I no tienen límite superior ni inferior, es decir no están acotados (unbounded) lo cual comporta que siempre puede haber un "más barato o no tan caro". Como se verá a continuación, este rasgo los distingue de la clase 2. Por ejemplo, se dice Pascual está triste, muy triste, más triste que Federico (no acotado), mientras esto sería difícil usar solo en el sentido de 'no acompañado' con estos modificadores: Pascual está solo, ?muy solo, "más solo que Federico (acotado).

Otra característica de los adjetivos escalares es que se organizan en pares antonímicos: caro es lo contrario de barato y rápido es lo contrario de lento, y cubren distintos segmentos de una escala que va entre dos polos. No se da en este caso una oposición exclusiva, sino que se sitúan por encima o por debajo de una norma o punto de referencia. El punto de referencia puede ser más o menos subjetivo y depende del contexto. Así, el enunciado:

(5) Ese chico es muy joven,

no se refiere a una edad objetiva, sino que puede interpretarse de distintas maneras según la intención comunicativa: "para casarse", "para viajar solo", "para haber escrito una novela premiada”, etc. Para los adjetivos guapo, feo no hay manera de medir el grado objetivamente, así el predicado extremadamente guapa se vale únicamente de la norma general conceptualizada por el locutor. Del mismo modo, una futbolista joven no tiene misma edad que un arzobispo joven, ya que se imponen distintas normas. 
Los pares de adjetivos polares no son totalmente equipolentes. El sesgo parece derivarse del hecho de que uno de los términos se valora de modo distinto. La ciudad no es muy (tan) fea no implica automáticamente una calificación positiva, ni es equiparable a Es hermosa o linda. Así pues, la relación polar no es la misma en todos los adjetivos escalares ya que depende del contenido semántico, de la perspectiva adoptada y ante todo de los objetos calificados y los contextos en que figuran.

Fundamentalmente, los adjetivos escalares son capaces de expresar “un más o menos”, es decir no suponen un límite máximo o mínimo. Son graduables mediante muy, un poco, extremadamente, etc. porque no tienen cotas (unbounded). Es inteligente se puede graduar sin problema con poco, muy, extremadamente, pero no va bien "completamente inteligente. Esta anomalía surge del hecho de que el Max exige un adjetivo que responda a otra conceptualización. Se debe a que ni inteligente ni otros adjetivos escalares que denotan cualidades están provistos de "un techo". Pasamos ahora a ver los adjetivos que corresponden a otro tipo de conceptualización.

\subsubsection{Clase 2}

La clase 2 abarca adjetivos que, como calvo, designan el punto final de un proceso culminado. Tienen la característica de ser polares, ya que los términos calvo - no calvo, vacío - lleno y roto - no roto son antonímicos y responden en principio a la idea de que alguien "es A o no es A”. En rigor, no existe en esta conceptualización ningún terreno intermedio, porque son estados, cualidades o condiciones que expresan puntos culminados. A diferencia de la clase $\mathrm{I}$, los adjetivos de límite son acotados (bounded) y en principio no permiten una graduación de tipo escalar. El siguiente esquema conceptual recoge estos aspectos:

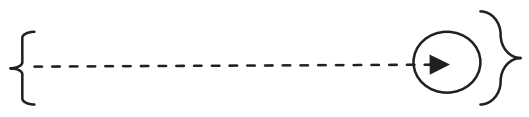

Los integrantes de esta clase son adjetivos y en eminente grado participios que focalizan un punto culminante, representado por el círculo de la Figura 2. Es verdad que estas palabras expresan grado, igual que los escalares, pero solo un grado máximo (o mínimo) que no se puede traspasar. La forma participal de un verbo perfectivo expresa de por sí la conclusión de un proceso o acción, hecho que los habilita para 
tomar modificadores de grado máximo. Se dice p.ej. completamente roto, totalmente destruido, enteramente inundado o absolutamente probibido.

Sin embargo, las conceptualizaciones no siempre son tajantes. Adjetivos como lleno, calvo, maduro, ciego, intacto y participios como roto, equivocado, deprimido, desteñido parecen indicar estados o condiciones absolutos que responden al esquema "sí o no". Pero esto solo vale desde un punto de vista lingüístico, o más bien lógico. Muchos de estos estados "finales" suponen procesos graduales que anteceden. Por este resquicio, entre lo conceptualizado como acotado y los procesos que se dan en la realidad, se hace posible una gradación de "más o menos" maduro, calvo, vacio, ciego, roto. De hecho, no sorprende oír decir frases como Pascual está muy calvo ya o Un poco calvo sí está o Pedro está más calvo que su padre, pues la calvicie se adquiere poco a poco. En otros casos, como en vivo - muerto, no parece haber posibilidad de indicar grados y se excluyen los modificadores escalares ${ }^{9}$

En los casos citados se impone una lectura gradual sobre un concepto que en principio es reacio a la gradación. Muy maduro, muy lleno implican que la madurez y la plenitud se conciben como estados que pueden ser "más o menos". De esta colisión pueden surgir efectos semánticos y estilísticos diversos, como se ve en estas frases:

(6) Cuidado, la caldera está muy llena.

(7) Estas ciruelas están muy maduras, ya no sirven para comer.

Como se ve, el modificador muy sirve aquí para advertir de que el objeto calificado ha sobrepasado un límite, que en principio no se puede sobrepasar. De ahí el valor negativo o ponderativo que se desprende de este empleo. No faltan a veces notas subjetivas al querer el hablante expresar una nueva visión de cierto estado de cosas.

Desde el punto de vista contrario, resulta interesante constatar que los típicos adjetivos escalares como alto, barato, inteligente no admiten fácilmente los Max. Frases como *Marcos es completamente inteligente $\mathrm{o}$ "Las naranjas están totalmente baratas son anómalas y reacias a la interpretación. Si los conceptos de inteligente y barato carecen de límite o punto culminante o, si se quiere, acotamiento (boundedness), no parece ser posible imponerles una conceptualización que satisfaga este requisito. Por si acaso, un adjetivo como perezoso da sentido en el enunciado Jorge es completamente perezoso, si 
se quiere expresar jocosamente que Jorge ha llegado al límite máximo de la pereza.

\subsubsection{Clase 3}

La clase 3 incluye adjetivos como excelente, maravilloso, falso, brillante, imposible, imprescindible, extraordinario, necesario, genial, que todos expresan algo extremo. La frase:

(8) ?La vista desde la terraza es muy maravillosa,

resulta tautológica porque el elemento superlativo está incluido en maravilloso. De la misma manera no se diría *un poco imposible, "extremadamente espantoso o "muy gigantesco, porque son combinaciones redundantes. Se concluye que estos adjetivos rehúyen una modificación de grado escalar porque ya en sí expresan un grado extremo ${ }^{\text {Io }}$. La misma contradicción se produce si se combina muy y -ísimo como en *Esta blusa es muy carísima. En cambio, los adjetivos/participios de esta clase se combinan fácilmente con los Max que simplemente subrayan que se ha alcanzado realmente el grado máximo:

(9) Esto es totalmente contradictorio, comentó el portavoz Julen Adrián. (CREA 2004: Prensa)

(г) No hay en esta creación, absolutamente genial, nada de elaborado: (CREA 200I: Gascón Soublette)

(i I) Sin embargo, la reacción ha sido acusarme de ser un agente de la CIA, lo cual es completamente falso. (CREA I984: Prensa)

Hay que agregar que, debido a la fluidez del sistema semántico, el uso de los Max no es totalmente unívoco con este tipo de adjetivos/participios. Al decir,

(I2) Juanín, estás terriblemente insoportable hoy, ¿qué te pasa?

el hablante logra un efecto comunicativo al romper con el esquema consabido: lo extremo no es suficiente sino que hay que añadir "un más”.

\subsection{Resumen}

De este examen se concluye que existen patrones relativamente fijos que regulan la modificación de grado de adjetivos/participios. El 
alcance de los Max queda delimitado con bastante claridad, según este esquema:

Tabla 2. Clases de adjetivos/participios y modificadores

\begin{tabular}{|c|c|c|c|}
\hline $\begin{array}{l}\text { Clase de } \\
\text { adjetivos }\end{array}$ & Combinación & $\begin{array}{l}\text { Clase de } \\
\text { modificadores }\end{array}$ & Ejemplos \\
\hline \multicolumn{4}{|l|}{$\begin{array}{l}\text { Adjetivos } \\
\text { graduables } \\
\text { plenos: }\end{array}$} \\
\hline $\begin{array}{l}\text { Adjetivos } \\
\text { graduables } \\
\text { (escalares) }\end{array}$ & $\longrightarrow$ & $\begin{array}{l}\text { Modificadores de } \\
\text { grado escalar }\end{array}$ & $\begin{array}{l}\text { muy alto, } \\
\text { extremadamente caro }\end{array}$ \\
\hline \multicolumn{4}{|l|}{$\begin{array}{l}\text { Adjetivos } \\
\text { graduables } \\
\text { limitados: }\end{array}$} \\
\hline $\begin{array}{l}\text { Adjetivos de } \\
\text { límite }\end{array}$ & $\longrightarrow$ & $\begin{array}{l}\text { Modificadores de } \\
\text { grado máximo }\end{array}$ & $\begin{array}{l}\text { completamente vacio, } \\
\text { totalmente roto }\end{array}$ \\
\hline $\begin{array}{l}\text { Adjetivos } \\
\text { extremos }\end{array}$ & $\longrightarrow$ & $\begin{array}{l}\text { Modificadores de } \\
\text { grado máximo }\end{array}$ & $\begin{array}{l}\text { totalmente imposible, } \\
\text { absolutamente necesario }\end{array}$ \\
\hline
\end{tabular}

Sin duda, la distribución recogida en la Tabla 2 es una idealización. Hasta cierto punto las categorías son permeables y permiten solapamientos. En primer lugar, los modificadores de la clase I pueden emplearse con muchos adjetivos/participios de las clases 2 y 3 . Sin embargo, parece ser que las restricciones del sentido inverso son más imperiosas.

Además, existen adjetivos/participios que admiten tanto una conceptualización gradual escalar y otra de límite. Los adjetivos sucio, limpio, borracho, falso se combinan con modificadores escalares y modificadores de grado máximo:

(I za) Pepito, vete al baño, estás totalmente sucio.

( $\mathrm{z}$ b) Jorge, la cocina está muy sucia, me das una mano.

(I4a) Felipe está completamente borracho

(I4b) Tu compañero está muy borracho, llévatelo a casa.

En estos casos y otros de traspaso de una clase a otra, el modificador imprime su sentido categorial a la cualidad o la propiedad denotada. 
En (I3 a) sucio se comporta como un adjetivo de límite, significando que "Pepe a llegado al límite máximo de suciedad". En el caso ( 13 b) se concibe el hecho de estar sucio como una propiedad que se puede graduar. Se debe notar que es el modificador el que imprime sus características sobre el adjetivo/participio modificado y no al revés.

En lo que sigue nos centraremos en los Max a fin de ver las distribuciones cuantitativas y las eventuales "atracciones" entre pares de modificador y modificado.

\section{Aproximación cuantitativa a los Max}

En base al inventario de ejemplos extraídos de CREA, mostramos a continuación una serie de tablas para hacer constar distintos sesgos distributivos. El total de ocurrencias de los cuatro Max en el período I 996-2000 (todos los medios) es de 8.408 ocurrencias. Como se ha indicado arriba (sección 3), la distribución cuantitativa tiene por fin ver la afinidad y solapamiento de los Max respecto a los lexemas modificados, en pocas palabras su grado de exclusividad o independencia. Ahora bien, como no existe ninguna medida previa de la intercambiabilidad de los Max (¿qué es mucho, qué es poco, qué es normal?), debemos conformarnos con hacer comparaciones internas. Por ejemplo, si absolutamente se comporta distribucionalmente de un modo diferente a los otros Max, esto es indicio de que su consonancia semántica con los lexemas modificados difiere de la de los otros modificadores. Se dirá que su atracción mutua tiene una motivación algo distinta.

Tabla 3. Frecuencia total de los Max en CREA.1996-2000

\begin{tabular}{lrrrrr}
\hline Max & Enteram. & Completam. & Totalm. & Absolutam. & Total \\
\hline Casos & 277 & $\mathrm{I} .834$ & 3.877 & 2.420 & 8.408 \\
\% del total & 3,3 & $2 \mathrm{I}, 8$ & $46, \mathrm{I}$ & 28,8 & $=100$ \\
\hline
\end{tabular}

Se ve que los Max se reparten de forma bastante desigual. La forma desfavorecida es enteramente que se usa casi I 4 veces menos que totalmente que está a la cabeza con 3.877 ocurrencias. En esta desproporción puede haber restricciones de registro e incluso distinciones semánticas. 
De este contingente de ocurrencias se han extraído los adjetivos/ participios compartidos por dos o más Max, que arrojan un total de I 20 lexemas:

Tabla 4. Lexemas modificados compartidos e índice de repeticiones de lexemas

\begin{tabular}{lrrrrr}
\hline Max & Enteram. & Completam. & Totalm. & Absolutam. & Total \\
\hline $\begin{array}{l}\text { Lexemas } \\
\text { compartidos }\end{array}$ & I 20 & I 20 & I 20 & I 20 & I 20 \\
\hline $\begin{array}{l}\text { Lexemas } \\
\text { repetidos }\end{array}$ & 84 & 50 & 50 & 59 & 243 \\
\hline $\begin{array}{l}\text { Total } \\
\text { ocurrencias }\end{array}$ & 204 & I70 & I70 & I79 & 723 \\
\hline $\begin{array}{l}\text { Indice de } \\
\text { repetición de } \\
\text { lexemas } \%\end{array}$ & 70,0 & 4 I, 6 & 4 I, 6 & 49,2 & \\
\hline
\end{tabular}

La Tabla 4 muestra el número de lexemas compartidos por los cuatro Max y usados una o más veces con los respectivos Max. Por ejemplo, para enteramente esto significa que de los I 20 lexemas compartidos hay 84 ocurrencias repetidas $(70,0 \%)$, lo que da un saldo de 204 ocurrencias en total. Esto quiere decir que enteramente, al ser el Max que más a menudo repite un lexema, tiene una tendencia a no salirse de sus linderos. Se puede concluir que enteramente está más circunscrito que los otros Max que tienen combinatorias más latas. Esto hay que apreciarlo a la luz de que los otros Max son entre 6 y I 4 veces más frecuentes que enteramente.

Otro aspecto afín es el grado de cobertura de dos maximadores, es decir la cantidad de veces que dos Max ocurren con el mismo lexema:

Tabla 5. Número y porcentaje de lexemas compartidos por dos Max

\begin{tabular}{|c|c|c|c|c|c|c|c|c|}
\hline \multirow[t]{2}{*}{$\operatorname{Max}$} & \multicolumn{2}{|c|}{ Enteram. } & \multicolumn{2}{|c|}{ Completam. } & \multicolumn{2}{|c|}{ Totalm. } & \multicolumn{2}{|c|}{ Absolutam. } \\
\hline & $\mathrm{N}^{\circ}$ & $\%$ & $\mathrm{~N}^{\circ}$ & $\%$ & $\mathrm{~N}^{\circ}$ & $\%$ & $\mathrm{~N}^{\circ}$ & $\%$ \\
\hline Enteram. & - & - & I 8 & I 5,0 & I7 & $\mathrm{I} 4,2$ & I 5 & I 2,5 \\
\hline Completam. & I 8 & I 5,O & - & - & 28 & 23,3 & I 8 & I 5,0 \\
\hline Totalm. & I7 & $\mathrm{I} 4,2$ & 28 & 23,3 & - & - & I 8 & I 5,0 \\
\hline Absolutam. & I 5 & I 2,5 & I 5 & I $5, O$ & I 8 & $\mathrm{I}_{5}, \mathrm{O}$ & - & - \\
\hline
\end{tabular}


En esta tabla se hace constar el número de lexemas compartidos por dos Max, es decir la cobertura entre los distintos modificadores. Así, en el renglón de enteramente se detalla en la columna de completamente la cantidad de lexemas distintos modificados por estos Max: las I 8 ocurrencias representan un I $5 \%$ de los I20 lexemas compartidos. El resultado muestra una leve polarización en el sentido de que enteramente y sobre todo absolutamente son más reacios al solapamiento que completamente y totalmente. Se debe concluir que absolutamente es algo más específico y no tan sustituible como lo son los otros Max. Esto vale también para enteramente, aunque en menor medida.

Otra forma de comprobar lo mismo es contabilizar el número de casos en que un Max es exclusivo, es decir no es compartido por otro ${ }^{\mathrm{II}}$ :

Tabla 6. Número de lexemas combinados exclusivamente con los respectivos Max y número de ocurrencias de estos lexemas

\begin{tabular}{|c|c|c|c|c|c|c|}
\hline Max & $\begin{array}{l}\mathrm{N}^{\circ} \text { de ocurrencias/ } \\
\text { lexemas }\end{array}$ & $\geq 5$ & 4 & 3 & 2 & $\begin{array}{l}\text { Total lexemas } \\
\text { distintos }\end{array}$ \\
\hline Enteram. & & I & I & I & I4 & I7 \\
\hline Completam. & & ○ & I & ○ & 4 & 5 \\
\hline Totalm. & & ○ & $\circ$ & ○ & 6 & 6 \\
\hline Absolutam. & & ○ & I & I & 8 & IO \\
\hline
\end{tabular}

Nuevamente se constata que enteramente y absolutamente son los Max que muestran más individualidad. En el caso de absolutamente hay ro lexemas distintos que son combinados exclusivamente con este Max. Este resultado da a entender que absolutamente despliega una semántica algo más particular que los otros.

\section{Perfil semántico de los Max}

Hay lugar para suponer que las distribuciones segadas de los Max están motivadas. Los casos de absolutamente y enteramente indican que su empleo es algo particular. Nuestra hipótesis es que los cuatro Max tratados, aunque en gran medida son reemplazables, conservan algo de su significado originario. Este significado nuclear explicaría que un Max particular atrae especialmente a cierta clase de adjetivos/participios. 
Las siguientes imágenes esquemáticas pretenden perfilar estos significados, destacando lo característico de cada Max:

Figura A. Entero

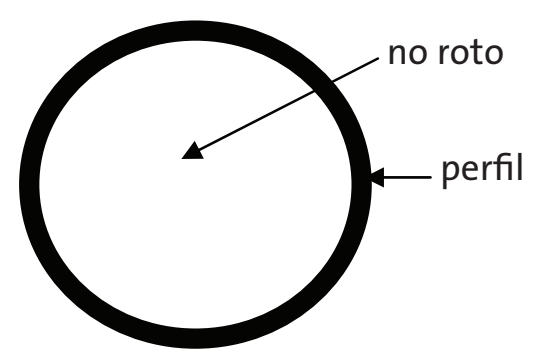

Entero pone de realce por un lado la integridad de un objeto o una masa delimitados, y por otro el hecho de que todas las piezas estén encajadas (contrario de roto).

Figura B. Completo

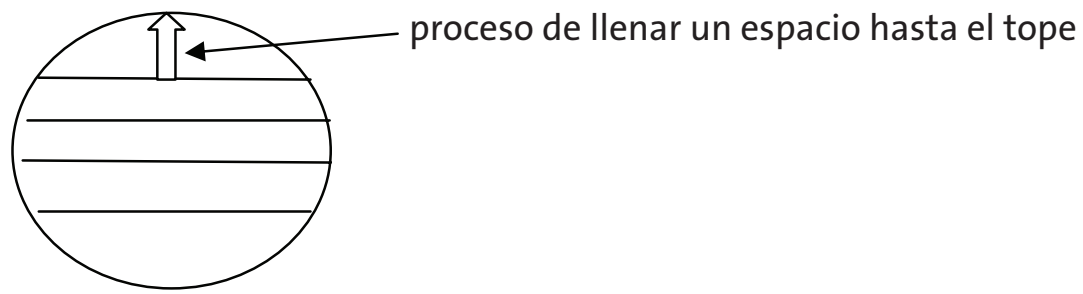

El perfil de completo parte del proceso de completar o completarse algo. Las piezas de la imagen tienden a rellenar todo el espacio hasta llegar a un límite. Cuando se dice que "la sala está completa", es que no hay espacio para más personas u objetos.

Figura C. Total



Total conlleva la idea de un proceso aditivo hasta llegar a un techo. El total de los gastos es la suma de los gastos particulares. Según esta perfilación, completo y total son muy afines, siendo el elemento aditivo más marcado en total. 
Figura D. Absoluto

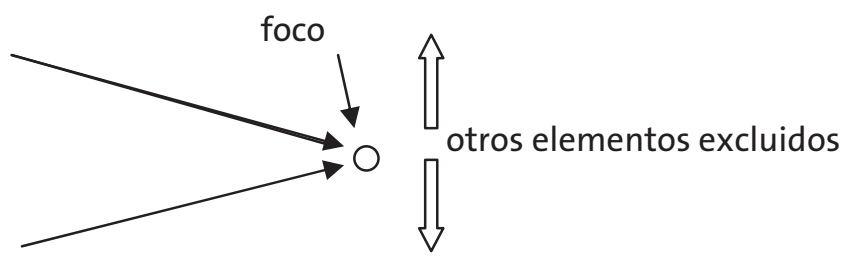

La configuración semántica de absoluto es, según nuestra hipótesis, distinta de la de los otros Max. La idea detrás de absoluto es la focalización en un punto preciso y extremo, excluyendo todo lo demás. Expresa así un valor máximo al que se llega no por agregación sino por exclusión.

\section{Aproximación cualitativa a los Max}

Como queda dicho, no es plausible que las distribuciones cuantitativas no encierren aspectos cualitativos. La primera constatación que se debe hacer es que los cuatro Max solo se construyen con adjetivos/participios de límite o de significado extremo o superlativo. En los materiales en que se basa este estudio no figuran con adjetivos/participios de carácter escalar.

La segunda constatación es que enteramente y absolutamente se desvían algo de la tónica de los otros dos. Como se ha visto, son los que se combinan con el mayor número de adjetivos/participios de forma exclusiva. Esto nos remite a la cuestión de saber si existe un motivo semántico de estas preferencias o si es debido al reducido tamaño del corpus o a otros factores. Si el sesgo distributivo que se ha comprobado se ve respaldado por los esquemas perfilados de los Max, hay lugar para creer que no es arbitrario sino debido a una atracción mutua.

Para dar una idea general de las combinatorias citamos a continuación I 5 combinaciones atestadas de los cuatro Max ${ }^{\mathrm{I} 2}$ :

Enteramente:

acristalado, activo, ajeno, automático, barroco, blanco, bueno, centralizado, cierto, controlado, conformado, correcto, condenable, dispuesto, descubierto, diverso

Completamente:

definido, descartado, destruido, diferente, distinto, dividido, dominado, enlodado, erótico, exento, falso, feliz, fiable, gratis, ignorado, ilegal 


\section{Totalmente:}

desairado, desprotegido, desamparado, detallado, distinto, dominado, ejecutado, equivocado, erróneo, exento, franco, formal, falso, globalizado, gratis

\section{Absolutamente:}

genuino, incapaz, inaceptable, inédito, ilegal, imprescindible, indispensable, injusto, irreal, inhábil, justo, justificable, legal, lineal, libre

Los casos citados dan una idea de la envergadura semántica de los adjetivos/participios de cada Max, pero no nos acerca a las "atracciones". Se debe recordar en primer lugar la laxitud del sistema, lo que queda demostrado por los adjetivos que ocurren con los cuatro Max: ajeno, distinto, falso, idéntico, legal ${ }^{\mathrm{I}}$. Ilustramos aquí estas posibilidades con el primer lexema citado ${ }^{\mathrm{I} 4}$ :

( 15$)$ [...] en el dominio de España por un pueblo enteramente ajeno y no cristiano de una religión hostil. (CREA I985: 3. J. Marías)

(I6) [...] como si se tratara de algo que a él y a mí nos resulte completamente ajeno. (CREA, I996-2000:IO. J.L. Méndez)

(17) Picasso, totalmente ajeno a esto, se centró en el aprendizaje de lo que le enseñaban, [...] (CREA 2002:Io. Prensa)

(I8) [...] probatorio de esto, que desde mi punto de vista es absolutamente ajeno a la cuestión que aquí se trata. (CREA I996-2000: 2. Oral)

No hay motivos para creer que hay una clara diferenciación semántica en estos casos. Sin embargo, si se pasa a los casos de uso exclusivo de un Max particular se entrevén algunos patrones. Miremos primero algunos ejemplos que juzgamos típicos de los respectivos Max:

(19) [...] los otros personajes no son ni enteramente buenos ni irrestrictamente malos. (CREA 2004:8)

(20) $[. .$.$] en especial el último [coche] cuya parte frontal quedó completa-$ mente destruida. (CREA 2004:7)

(2I) -Claro, por eso se me acercan tipos equivocados, totalmente banales, que están para el físico y nada más. (CREA 2004: I4)

(22) [...] otra forma del espectro de la Leismaniasis es absolutamente necesaria para el estudio del efecto inmunoterapéutico (...) (CREA 2004:29) 
(23) Cuando tenía I8 años recuerdo haber dicho algo absolutamente impensable en un chico con esa edad, (...) (CREA 2004:60)

No cabe duda de que en estos contextos hubiera sido posible emplear los Max de modo diferente: en (20) sería natural decir enteramente o totalmente destruida y en (2 I) convendrían completamente o absolutamente. Aun siendo así, puede haber en estos ejemplos alguna atracción semánticamente motivada. En el caso de enteramente (I9) apunta la idea de una propiedad que engloba varios aspectos o partes. Como se ha visto, entero subraya esta integridad de una superficie o un objeto, mientras que completamente y totalmente perfilan el tope de algo sumado.

Los casos más particulares son aquellos en que figura absolutamente. En (22) y (23) se predican situaciones modales extremas: algo necesario y algo impensable. Como se ha hipotetizado, absoluto enfoca un punto excluyendo otras posibilidades; así, es natural que este Max sea adecuado en contextos negativos y extremos. A diferencia de completamente y totalmente que construyen una totalidad, absolutamente señala y refuerza lo que en sí es extremo. Se ha verificado en los materiales que absolutamente se combina ante todo con adjetivos extremos y negativos. En los casos modificados por absolutamente son abundantes los adjetivos que empiezan por in-o im-: incapaz, inaceptable, inédito, ilegal, imprescindible, indispensable, incoherente, inconstitucional, etcétera, hasta 30 lexemas distintos.

A continuación se presenta otra forma de comprobar diferencias entre los Max. Partiendo de todas las ocurrencias en CREA de adjetivos que hipotéticamente deben combinarse con determinados Max, se podrán detectar las líneas de fuerza del sistema. Las siguientes catas contienen adjetivos extremos de diversa semántica (negativos y superlativos) con el supuesto de que deben ser atraídos sobre todo por absolutamente. Los casos atestados deben contrastarse con la ocurrencia relativa de los cuatro Max: enteramente 3,3\%, completamente $2 \mathrm{I}, 8 \%$, totalmente $46, \mathrm{I} \%$ y absolutamente $28,8 \%$ (basado en 8.408 ocurrencias).

Las cifras de esta prueba son variables pero hablan por sí mismas. Absolutamente es el Max claramente favorecido y la diferencia llega a ser realmente contundente con indispensable (típicamente negativo) y maravilloso (típicamente superlativo) donde es el único Max atestado. No cabe duda de que absolutamente se liga con esta clase de adjetivos en consonancia con la hipótesis acerca de su semántica. 
Tabla 7. Colocaciones de los Max con adjetivos negativos y superlativos (CREA íntegro)

\begin{tabular}{|c|c|c|c|c|c|}
\hline \multirow[t]{2}{*}{$\operatorname{Max}$} & \multicolumn{5}{|c|}{$\mathrm{N}^{\circ}$ de casos } \\
\hline & $\begin{array}{l}\text { indispens- } \\
\text { able }\end{array}$ & $\begin{array}{l}\text { extraor- } \\
\text { dinario }\end{array}$ & imposible & $\begin{array}{l}\text { mara- } \\
\text { villoso }\end{array}$ & fantástico \\
\hline enteramente & ० & o & 3 & ० & o \\
\hline completamente & 3 & o & 20 & ० & I \\
\hline totalmente & o & I & 37 & ० & o \\
\hline absolutamente & 48 & 5 & 88 & 8 & 2 \\
\hline
\end{tabular}

En resumen, no parece haber una atracción mutua exclusiva entre los respectivos Max y grupos de adjetivos/participios. En un material de mayor volumen habría seguramente un solapamiento aún mayor, pero igualmente se destacarían tendencias, tal como se ha comprobado con absolutamente. Recogiendo integralmente en CREA todas las ocurrencias de algunos de los adjetivos registrados con los cuatro Max, a saber ajeno, distinto, falso, ilegal, se comprueban tendencias y preferencias, pero no lo bastante claras como para hablar de colocaciones fijas y exclusivas de este Max. Queda por hacer más pesquisas para descubrir y analizar estas pautas. En todo caso, la distribución de los Max tal como se aprecia en la Tabla 8 , no se corresponde con las frecuencias relativas de la totalidad de ocurrencias ${ }^{15}$ :

Tabla 8. Colocaciones de los Max con cuatro adjetivos (CREA íntegro)

\begin{tabular}{lrrrr}
\hline \multirow{2}{*}{ Max } & \multicolumn{4}{c}{$\mathrm{N}^{\circ}$ de casos } \\
\cline { 2 - 5 } & ajeno & distinto & falso & ilegal \\
\hline enteramente & 3 & $\mathrm{I}$ & 3 & $\mathrm{I}$ \\
completamente & 39 & $\mathrm{I} 46$ & 20 & 8 \\
totalmente & 62 & $\mathrm{I} 2 \mathrm{I}$ & $6 \mathrm{I}$ & $\mathrm{I} 3$ \\
absolutamente & 8 & $\mathrm{I} 9$ & 94 & $\mathrm{I} 3$ \\
\hline
\end{tabular}

En general, las proporciones siguen las tendencias generales de los Max con dos claras excepciones de sobreuso: absolutamente falso y absolutamente ilegal. 


\section{Conclusiones}

Hay que reconocer que este estudio no proporciona respuestas unívocas a las hipótesis. Si bien hay una atracción mutua entre los Max y grupos particulares de adjetivos/participios, no parece haber mucha atracción exclusiva entre los respectivos Max y los elementos modificados. Lo más llamativo es que los Max estudiados aquí se solapan en gran medida. Se ha verificado que los Max solo seleccionan los adjetivos/participios de límite y extremos o superlativos. No figura ningún adjetivo típicamente escalar como alto-bajo o caro-barato en los materiales.

Se ha visto que el índice de cobertura entre dos Max y los lexemas atestados (es decir, el número de lexemas compartidos) varía entre un I $2,5 \%$ y un $23,5 \%$. Es difícil sacar conclusiones claras de estas cifras, dado el volumen relativamente limitado del corpus. El porcentaje de 23,5 es, sin embargo, apreciable, pues casi cada cuatro lexema del corpus ocurre tanto con completamente como con totalmente. Resulta, pues, que estos dos modificadores tienen aire de familia y se comportan de forma casi igual. Si se ampliara el corpus, con toda probabilidad la cobertura sería aún mayor.

Enteramente, y sobre todo absolutamente, parecen desviarse un poco de los otros dos. Al parecer, entero rastrea su significado original al poner de relieve la idea de "que concierne a todos los aspectos que conforman algo en su integridad". Si se dice El cuadro estaba enteramente destrozado o El niño estaba enteramente sucio, lo que se representa es algo que engloba a todas las partes. En cambio, El niño estaba totalmente sucio más bien evoca el grado máximo de suciedad.

Absolutamente, a su vez, se ve atraído por adjetivos/participios que denotan algo extremo y negativo, que vemos como un efecto de su función como focalizador. Parece posible ver en esto una consonancia con el perfil semántico originario de absoluto que no se basa en la imagen de algo sumado o colmado como completamente y totalmente, sino en lo máximamente exclusivo. Adjetivos como indispensable, inédito y muchos otros con el prefijo in- (im-) son ejemplos típicos de las colocaciones de absolutamente.

Así pues, los materiales dejan entrever ciertas combinatorias que parecen ser motivadas pero, a la luz de que ajeno, falso, distinto, idéntico figuran con los cuatro Max, es mejor no hablar de restricciones semánticas. Se trata de avenencias y tendencias. Las restricciones se dan ante todo con respecto otros modificadores de grado, no en el interior del grupo de los Max. 


\section{Notas}

I. Ver infra, ap. 4, p. I76.

2. "Atracción mutua” es una expresión preteórica que simplemente alude al hecho de que un Max particular se combina con cierto tipo de adjetivos/participios (+atracción), antes que con otros tipos de adjetivos/participios (-atracción).

3. Las notaciones ?, ??, * indican grados de anomalía semántica, no una restricción sintáctica. Esto quiere decir enunciados marcados como más o menos anómalos pueden encontrarse en la lengua, pero se trata en este caso de explotar el sistema para obtener efectos estilísticos, jocosos o hiperbólicos.

4. Este aspecto se tratará somera e indirectamente.

5. En CREA (íntegro) enteramente imposible arroja 3 ejemplos, mientras que absolutamente imposible figura 88 veces.

6. CREA es el acrónimo por El Corpus de Referencia del Español Actual, elaborado por la Real Academia Española, consta de I 54.279.050 formas y cubre el período entre I975 y 2004.

7. Frío puede reanalizarse como un adjetivo acotado si refiere al proceso de enfriarse, como en la frase El cadáver está completamente frío.

8. Esta clasificación sigue de cerca la que presenta Paradis (I997:49).

9. Evidentemente, en sentido figurado estos adjetivos/participios son perfectamente graduables: Marta es muy viva y Pedro es más muerto que el tronco de un árbol.

IO. Remitimos a la presentación de Paradis (I997: 54 y sigs.).

I I. Este recuento se basa en otro inventario extraído de CREA I996-2000 que consta de 200 ejemplos de cada Max.

I2. Se citan los lexemas en masculino singular.

I3. La cobertura sería decididamente mayor si el estudio se basara en materiales más extensos.

I4. En el caso de enteramente el ejemplo proviene del año I985.

I 5. Cf. supra, tabla 3, p. I74.

\section{Referencias}

Allerton, David J. I987. "English intensifiers and their idiosyncrasies". In: Steele, Ross \& Terry Threadgold. Language topics. Essays in honour of Michael Halliday, 2. I 5-3 I. Amsterdam: John Benjamins. 
Bierwisch, Manfred. I967. "Some semantic universals of German adjectives". Foundations of Language 3. I-36.

Bolinger, Dwight. I972. Degree words. The Hague: Mouton.

Cruse, D. Alan. 1986. Lexical Semantics. Cambridge: Cambridge University Press.

Cruse, D. Alan \& Pagona Togia. I996. "Towards a cognitive model of antonymy". Journal of lexicology I. I I3-I4I.

Cuenca, Maria Josep \& Joseph Hilferty. I999. Introducción a la lingüística cognitiva. Barcelona: Ariel Lingüística.

Erman, Britt. 2009. Is there such a thing as a random combination? A usagebased study of specific construals as apparent in adverb-adjective combinations. Stockholm: Dept. of English, Stockholm University. (manuscrito)

Goldberg, Adele E. I995. Constructions: A Construction Grammar approach to argument structure. Chicago: University of Chicago Press.

Leech, Geoffry. I974. Semantics. Hammondsworth: Penguin Books.

Leonetti, Manuel. 2007. Los cuantificadores. Madrid: Arco Libros.

Moreno Teva, Inmaculada. 20I 2. Las secuencias formulaicas en la adquisición de español L2. Tesis doctoral. Stockholm, Universidad de Estocolmo.

Paradis, Carita. I 997. Degree modifiers of adjectives in spoken British English. Tesis doctoral. Lund: Lund University Press.

Real Academia Española. 20ıо. Nueva Gramática de la Lengua Española. Manual. Madrid: Espasa Libros.

Wray, Alison. 2002. Formulaic Language and the Lexicon. Oxford: Oxford University Press.

Östman, Jan-Ola \& Mirjam Fried. 2005. "The cognitive grounding of Construction grammar”. In: Östman, Jan-Ola \& Mirjam Fried. Construction grammars. Cognitive grounding and theoretical extensions. Amsterdam \& Philadelphia: John Benjamins. I-I3. 